\title{
La relación entre el aprendizaje léxico y el desarrollo gramatical
}

\author{
Elisabet Serrat ${ }^{1}$, Mònica Sanz-Torrent ${ }^{2}$, Iris Badia ${ }^{2}$, Eva \\ Aguilar $^{3}$, Raquel Olmo $^{1}$, Fernanda Lara $^{4}$, LlORENÇ ANDreU $^{2,5}$ \\ Y MIQUEL SERRA ${ }^{2}$ \\ ${ }^{1}$ Universitat de Girona; ${ }^{2}$ Universitat de Barcelona; ${ }^{3}$ Universitat Illes Balears; \\ ${ }^{4}$ Universidad Nacional de Colombia; ${ }^{5}$ Universitat Oberta de Catalunya
}

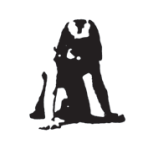

\section{Resumen}

El presente trabajo se centra en estudiar la relación que existe entre el desarrollo de léxico y el de la morfosintaxis. Concretamente pretendemos explorar el tipo de vocabulario que mejor predice el desarrollo de la morfología verbal y el de la complejidad gramatical, así como establecer el tipo de relación entre desarrollo léxico y desarrollo morfosintáctico. La muestra comprende 517 niños de edades comprendidas entre los 18 meses y los 30 meses. Los datos se han recogido a partir de la adaptación al catalán del instrumento MacArthur-Bates Communicative Development Inventories (CDI). Los resultados muestran que el mejor predictor del desarrollo morfológico y gramatical es el vocabulario de clase cerrada, conjuntamente con el vocabulario general. Por otra parte, se observa una relación predominantemente lineal entre el desarrollo del léxico y el desarrollo morfosintáctico.

Palabras clave: Desarrollo léxico y gramatical, adquisición del lenguaje, MacArthur-Bates Communicative Development Inventories (CDI).

\section{The relationship between lexical learning and grammatical development}

\begin{abstract}
The present study focuses on the relationship between lexical and morphosyntactic development. Specifically, we intend to explore which kind of vocabulary best predicts the development of verbal morphology and grammatical complexity, and to analyse the relationship between lexical and morphosyntactic development. The sample was composed of 517 children aged from 18 months to 30 months. The data were collected by means of the Catalan adaptation of MacArthur-Bates Communicative Development Inventories (CDI). The results show that the best predictor of morphological and grammatical development is closed class vocabulary, together with general vocabulary. On the other hand, a predominantly linear relationship between lexical development and morphosyntactic development is observed.
\end{abstract}

Keywords: Lexical and grammatical development, language acquisition, MacArthur-Bates Communicative Development Inventories (CDI).

\footnotetext{
Agradecimientos: La redacción de este artículo se ha realizado al amparo de los proyectos SEJ2006-12039/PSIC, SEJ200762743, SEJ2007-65236, SEJ2006-12616. La recogida de datos ha sido posible gracias a la colaboración de muchos padres y madres de niños de las escuelas participantes. Queremos agradecer especialmente la ayuda de: O. Fernández, E. Ferrer, R. Lluent, A. Mallat, E. Noguera, A. Novella, J. Serrano, E. Teruel y F. Trullàs. Finalmente, los autores agradecen los comentarios de dos revisores anónimos que han contribuido a mejorar notablemente el manuscrito original. Correspondencia con los autores: Mònica Sanz Torrent. Departament de Psicologia Bàsica. Universitat de Barcelona, Vall d'Hebrón, 17108035 Barcelona. Tel: +34933125150 Fax: +3493 4021363. E-mail: monicasanz@ub.edu
} 


\section{Introducción}

El proceso de adquisición de los componentes del lenguaje durante las primeras etapas del desarrollo ha sido estudiado y descrito fundamentalmente en cuanto a su progreso de forma aislada. Sin embargo, estudiar el desarrollo de los componentes del lenguaje independientemente no proporciona una explicación adecuada de cómo transcurre la adquisición del lenguaje, puesto que los diversos componentes del sistema lingüístico no son independientes entre ellos. Solamente a través del estudio de la interacción entre los diferentes niveles del conocimiento lingüístico -conocida como interface- podremos avanzar en el conocimiento de cuestiones fundamentales acerca del desarrollo del lenguaje (Serra, 2008). Precisamente, algunas propuestas explicativas sobre los mecanismos de adquisición gramatical se basan en que un componente se desarrolla en dependencia con otro. De esta consideración se derivan la propuesta del bootstrapping sintáctico (Fisher, Hall, Rakowitz y Gleitman, 1994; Gleitman, 1990; Naigles, 1996; Naigles y Hoff-Ginsberg, 1995) y también la propuesta del bootstrapping semántico (Bowerman, 1988; Goldberg, 1995; Goldberg, Casenhiser y Sethuraman, 2004; Grimshaw, 1981; Pinker, 1984, 1989).

Actualmente, sin entrar en la discusión acerca de los mecanismos de bootstrapping, pero sí dentro del estudio de esa interacción entre niveles lingüísticos, una de las cuestiones centrales es la de las relaciones entre el desarrollo léxico y el gramatical. En esta línea, se considera esencial el estudio de la estructuración del léxico o la emergencia de la gramática a través de fenómenos como la gramaticalización de nombres y verbos. Varios trabajos han aportado datos sobre esta cuestión y han mostrado una relación no lineal entre el léxico inicial y los inicios del desarrollo gramatical en lengua inglesa (Bates y Goodman, 1997; Dromi, 1987; Marchman y Bates, 1994). Este patrón de desarrollo también se ha observado en estudios en otras lenguas, así por ejemplo, para el italiano (Caselli, Casadio y Bates, 1999), el hebreo (Maital, Dromi, Sagi y Bornstein, 2000), el islandés (Thordardottir, Ellis Weismer y Evans, 2002), el catalán (Serra y Sanz-Torrent, 2004) o el francés (Bassano, 2000). A partir de estos datos, se sugiere, no sólo que el crecimiento del vocabulario precede al desarrollo gramatical, sino también que los avances en el componente gramatical se explican a partir de los cambios que se observan en el desarrollo del léxico.

Para explicar este fenómeno se han defendido diversos puntos de vista. Una de las propuestas asume que la gramática emerge a partir de los mecanismos implicados en la adquisición del léxico. Bajo esta hipótesis, mientras el léxico aumenta en tamaño y en especialización semántica, la gramática deviene organizada de manera cada vez más compleja. Es decir, las formas gramaticales serían propiedades emergentes de un sistema lingüístico unificado y estarían fuertemente ligadas a los cambios en el léxico dentro de este sistema. En tanto que la medida y organización del léxico crece, la calidad de la gramática emergente cambia. En este caso se requeriría una 'masa crítica' de vocabulario para desarrollar un nivel particular de complejidad gramatical (Bates y Goodman, 1997; Marchman y Bates, 1994; Plunkett y Marchman, 1993).

Desde otra perspectiva, asociada a las teorías "usage based" (Tomasello, 2003), se sostiene que las primeras combinaciones de palabras son muy rutinizadas y específicas de una situación, hecho que conlleva una organización de complejidad creciente, primero de tipo monótonica o lineal, para pasar a ser no-lineal a partir de un cierto nivel de masa crítica o complejidad, que se concreta en el componente gramatical. Aprender gramática, como aprender palabras, puede venir dado por procesos que son específicos de ítems concretos y dependientes de la frecuencia. Solamente a partir del aprendizaje de un léxico crecientemente especializado e interrelacionado, las estructuras gramaticales devienen codificadas en términos de su forma sintáctica abstracta (Akhtar, 1999; Braine, 1976; Lieven, Pine y Baldwin, 1997; Tomasello, 2003). 
Estas propuestas asumen una asociación fuerte entre los principios de aprendizaje implicados en la adquisición léxica y la gramatical y son compatibles con la idea de una integración del componente léxico-semántico y de la gramática, cada vez más presente en las teorías lingüísticas (por ej., Goldberg, 1999).

Además, dentro de las relaciones entre el léxico y la gramática, previamente conviene establecer si dichas relaciones se basan en aspectos generales del desarrollo conceptual o bien específicos de la forma, es decir, de la lengua concreta. Dos estudios fundamentales examinan esta cuestión en niños bilingües inglés-español: tanto el trabajo de Conboy y Thal (2006) como el de Marchman, Martínez-Sussmann y Dale (2004) estudian si las habilidades gramaticales dependen del desarrollo léxico estrictamente en una lengua o del desarrollo del vocabulario conceptual a través de diversas lenguas. Los resultados de ambos estudios muestran que las relaciones que se dan en niños bilingües entre desarrollo léxico y gramatical son específicas para cada lengua, y que no se da una relación entre desarrollo léxico conjunto (ya sea léxico conceptual o léxico combinado) de ambas lenguas y desarrollo gramatical en cualquiera de ellas.

También conviene apuntar que recientemente se ha discutido que la relación entre vocabulario y gramática sea no lineal. La aportación de Dixon y Marchman (2007) es especialmente relevante para esta cuestión, ya que estos autores defienden que la relación entre el léxico y la gramática puede existir, pero que ésta es sincrónica y mutua, de la misma manera que observaron Anisfeld, Rosenberg, Hoberman y Gasparini (1998). Una relación sincrónica entre los componentes lingüísticos en desarrollo puede hacer pensar en que no existe relación entre ellos, pero como dichos autores hacen notar (Dixon y Marchman, 2007), el hecho de que la relación sea sincrónica no implica que la gramática no dependa del léxico o que no sea necesaria una determinada masa crítica.

Por otra parte, también hay que tener en cuenta que la relación que estamos comentando es la que se ha hallado entre la cantidad de vocabulario total y los inicios gramaticales, a menudo medidos a partir de la complejidad sintáctica general. Es posible que los mecanismos que hay bajo los efectos de la masa crítica se expliquen mejor en términos de relaciones entre logros de léxico específico y la emergencia de patrones de abstracción precisos que formen la base de regularidades gramaticales. Por ejemplo, podría ser que el uso del tiempo pasado en inglés fuera más dependiente del aprendizaje de ítems léxicos particulares (los verbos) que proporcionaran las claves relevantes a partir de las cuales abstraer el patrón de pasado regular, que del vocabulario total.

En este sentido, los trabajos de Marchman y Bates (1994) y Plunkett y Marchman (1993) se centraron en las relaciones entre el número de formas verbales regulares y el uso productivo de la flexión de pasado. Así, por ejemplo, en lengua inglesa, Marchman y Bates (1994) mostraron que el desarrollo de la marca morfológica del pasado verbal se relacionaba de manera no lineal con el tamaño de vocabulario verbal. En este estudio el tamaño del vocabulario se mostró como un claro predictor del uso de las formas verbales regulares en inglés, correlacionando más fuertemente que la edad cronológica de los niños. Por otra parte, Caselli et al. (1999) presentaron un estudio comparativo entre la lengua inglesa y la italiana -lengua románica con mayor morfologización nominal y verbal. En sus datos observaron una interrelación en la complejidad gramatical y el tamaño del vocabulario muy parecido en las dos lenguas. En la misma línea, Thordardottir et al. (2002) obtuvieron resultados tanto en lengua inglesa como islandesa, enfatizando que en la lengua morfológicamente más rica, la islandesa, se necesitaba una mayor masa crítica léxica para el inicio del desarrollo morfológico. También Serrat, Sanz-Torrent y Bel (2004) observan que una determinada masa léxica verbal precede la aceleración de aprendizaje morfológico, aunque la relación se muestra continua entre ambos componentes.

Precisamente, en la línea de los estudios que acabamos de comentar, tanto Bates y Goodman (1997) como Dixon y Marchman (2007), señalan que sería interesante ir más allá de medidas léxicas globales e indagar qué aspectos o características específicos del 
conocimiento léxico infantil sirven (o no sirven) de precursores de la abstracción gramatical de regularidades gramaticales específicas.

Teniendo en cuenta la sugerencia de los autores referidos, en este estudio se pretende explorar la interrelación entre el léxico inicial y el desarrollo gramatical del lenguaje en algunos aspectos específicos. Hasta el momento, solamente el trabajo reciente de Stolt, Haataja, Lapinleimu y Lehtonen (2009), con niños finlandeses de 2 años de edad, retoma esta línea de investigación y aborda la cuestión de la relación entre medidas de vocabulario específicas y la emergencia de aspectos gramaticales en relación con la flexión nominal y verbal.

El presente estudio emplea informes paternos en lengua catalana para la recogida de los datos. En primer lugar se efectuará una descripción general del desarrollo de la morfología verbal y de la complejidad gramatical en relación con la edad y con la masa léxica del vocabulario general, nominal, verbal y de clase cerrada. En segundo lugar, se pretende relacionar el nivel de vocabulario general, verbal, nominal y de clase cerrada con el desarrollo morfológico verbal y con la complejidad gramatical, que es el objetivo principal de la investigación. A partir de estos datos, podremos establecer si se da linealidad o no entre los componentes analizados. Además, también podremos determinar cuál es la masa léxica, general o específica, necesaria para observar cambios concretos, si los hay, en el desarrollo morfosintáctico y conocer qué tipo de léxico predice mejor dicho desarrollo.

\section{Metodología}

\section{Participantes}

La muestra de este estudio está compuesta por un total de 517 sujetos de lengua materna catalana de edades comprendidas entre 16 y 30 meses. El muestreo se realizó en distintas zonas, urbanas y rurales, que tienen el catalán como lengua familiar. En Cataluña se aplicó por provincias con el fin de obtener una muestra representativa de la distribución real de población por zona territorial. Asimismo, se obtuvieron datos en las Islas Baleares en función de su peso de población. Además también hay una parte de los datos que proceden del País Valenciano y de Andorra. Respecto a las características lingüísticas, sólo se incluyeron los sujetos que presentaban la lengua catalana cómo familiar, entendiéndose así que los dos padres, o bien uno de ellos, usara únicamente la lengua catalana para dirigirse a su hija o hijo. Se excluyeron de la muestra los niños/niñas que presentaban deficiencia cognitiva, complicaciones médicas severas o prematuridad importante -el punto de corte se estableció a las 36 semanas de edad gestacional. La tabla I presenta la distribución de la muestra en función de la edad y del sexo de los sujetos.

\section{Materiales}

Los datos fueron obtenidos mediante la aplicación de los cuestionarios "MacArthurBates Communicative Development Inventories" (CDIs), específicamente el cuestionario CDI-II (Word and Sentences), adaptado previamente a la lengua catalana y llamado "MacArthur-Bates Inventari del Desenvolupament de les Habilitats Comunicatives, adaptaciò al català" (Serrat et al., 2005) y actualmente en proceso de normativación.

La versión catalana se ha diseñado para su aplicación mediante dos cuestionarios para niños desde 8 meses hasta a 30 meses de edad. Por un lado, el Inventario de palabras y gestos para niños de edades comprendidas entre los 8 y los 18 meses (conocido como CDI-I) y, por el otro, una segunda versión denominada Palabras y Oraciones que está dedicada a niños entre 16 y 30 meses (Conocido como CDI-II).

Los inventarios incluyen un gran número de preguntas o ítems que señalan o completan los padres. De este modo, el CDI-I presenta dos partes, la primera dedicada a las 
TABLA I

Distribución de la muestra por edad y sexo

\begin{tabular}{cccc}
\hline Edad & Niñas & Niños & Total \\
\hline $1 ; 4$ & 12 & 6 & 18 \\
$1 ; 5$ & 12 & 8 & 20 \\
$1 ; 6$ & 11 & 19 & 30 \\
$1 ; 7$ & 15 & 16 & 31 \\
$1 ; 8$ & 17 & 16 & 32 \\
$1 ; 9$ & 16 & 8 & 24 \\
$1 ; 10$ & 19 & 16 & 35 \\
$1 ; 11$ & 28 & 21 & 49 \\
$2 ; 0$ & 27 & 27 & 54 \\
$2 ; 1$ & 20 & 23 & 43 \\
$2 ; 2$ & 24 & 17 & 41 \\
$2 ; 3$ & 21 & 12 & 33 \\
$2 ; 4$ & 18 & 22 & 40 \\
$2 ; 5$ & 16 & 10 & 26 \\
$2 ; 6$ & 26 & 14 & 40 \\
Total & 282 & 235 & 517 \\
\hline
\end{tabular}

primeras palabras permite recoger información sobre diferentes aspectos pues incluye cinco subapartados: "Primeras muestras de comprensión", "Frases", "Maneras de hablar", "Lista de vocabulario" y "Primera Palabra". La lista de vocabulario recoge 19 categorías: interjecciones y sonidos de animales y cosas, animales, vehículos, alimentos, juguetes, ropa y piezas de vestir, partes del cuerpo, muebles, objetos, lugares, gente y personas, juegos y rutinas, acciones y procesos, tiempo, cualidades y atributos, pronombres, preguntas, preposiciones, locativos y modo y cuantificadores y artículos. En la segunda parte, se recoge información sobre gestos y acciones y consta de seis subapartados: "Primeros gestos", "Juegos con adultos y rutinas", "Acciones con objetos", "Jugando a ser adulto", "Imitaciones de otras acciones de los adultos", "Objetos que se usan en lugar de otro" (Juego simbólico).

El CDI-II, por su lado, para niños de 16 a 30 meses, incluye un listado de vocabulario compuesto por un total de 678 ítems agrupados en 22 categorías distintas. Además, el cuestionario contiene varios apartados referidos a la producción de la morfología nominal y verbal, así como a aspectos sintácticos. Concretamente, incluye una primera parte denominada "El uso de las palabras" que cuenta con un primer subapartado dedicado a la producción de la primera palabra y seguidamente la lista de vocabulario compuesto por las veintidós categorías, diecinueve que se recogen en la versión anterior pero ampliando en tres el número de categorías léxicas. El segundo subapartado, bajo el epígrafe "Como usa y entiende el niño/a el lenguaje", recoge diversos aspectos de la comprensión y uso del lenguaje relacionados con la memoria, la permanencia del objeto, la posesión, la comprensión de órdenes, etcétera, con un total de 5 preguntas con tres tipos de respuesta ("todavía no", "a veces", "muchas veces"). La segunda parte, esta dedicada a "Oraciones y Gramática" y comprende cinco subapartados: sobre terminaciones de las palabras, formas verbales irregulares, palabras sorprendentes (sobre-regulaciones), combinación de palabras y complejidad de frases.

En la bibliografía sobre la adquisición del lenguaje se considera que los informes parentales son fiables y representativos de las capacidades lingüísticas de los niños y niñas (Bates, Bretherton y Snyder, 1988; Fenson et al., 2007) y varios estudios con este instrumento en lenguas cercanas al catalán así lo han mostrado: Thal, Jackson-Maldonado y Acosta (2000) para el español, y Pérez Pereira y Resches (2007, 2008) para el gallego. Es de especial interés para el presente estudio el trabajo de Thal et al. (2000) puesto que estas autoras muestran, utilizando los cuestionarios MacArthur-Bates en su 
versión mexicana, que los padres son capaces de diferenciar entre vocabulario y gramática. Además, es importante precisar que las escalas MacArthur-Bates presentan ventajas sobre otros instrumentos de recogida de datos. En primer lugar, permiten evaluar el desarrollo comunicativo y lingüístico en muestras de sujetos muy amplias y, en segundo lugar, facilitan la realización de estudios comparativos debido a la existencia de adaptaciones en varias lenguas. En el estado español se ha adaptado al gallego (Pérez-Pereira y García-Soto, 2003); al castellano (López-Ornat et al., 2005) y al euskara (Barreña et al., 2008; García, Arratibel, Barreña, Ezeizabarrena, 2008). En castellano también existe una adaptación para niños con síndrome de Down (Galeote et al., 2006). La versión en catalán del CDI está basada en la versión original americana (Fenson et al., 1993), así como en las versiones gallega (Pérez-Pereira y García Soto, 2003), española-mexicana (Jackson-Maldonado et al., 1993) y española-castellana (López-Ornat et al., 2005).

\section{Tratamiento de los datos}

Para el análisis de datos se crearon las siguientes variables, agrupando categorías y/o ítems tal como se describe a continuación:

El índice de masa léxica (ML) incluye todas las categorías léxicas (nombres comunes, nombres propios, verbos, adjetivos, adverbios, etcétera), así como también las interjecciones, sonidos de animales y cosas, las fórmulas sociales y las rutinas. La puntuación máxima que pueden obtener es 678. Si subdividimos esta masa en nombres propios y comunes, tenemos el índice de masa léxica nominal (MLN). En este caso, la puntuación máxima puede ser de 363. El índice de masa léxica verbal (MLV) únicamente incluye los ítems del apartado "Acciones, procesos y estados" del cuestionario, que recoge verbos y su puntuación máxima es de 103 . Por otra parte, el índice de masa léxica de categoría cerrada (MLC) incluye la suma de los ítems de los apartados de "Preguntas", "Pronombres y determinantes posesivos y demostrativos", "Auxiliares", "conectivas" y todos los ítems de los apartados "Preposiciones, locativos y modo" y "Cuantificadores y artículos” excepto los adverbios. La puntuación máxima que se puede obtener es 90 . Por otro lado, para calcular la "Morfología verbal” se realizó la suma de las puntuaciones 0,1 y 2 -correspondientes a "todavía no", "a veces”, "Muchas veces”- de las preguntas 5 a 10 que se refieren a distintos tiempos verbales (por ejemplo, la pregunta 8 se refiere a si el niño produce formas de pasado o la 9 a formas de futuro). A esta puntuación se sumaron las puntuaciones de los ítems (correspondiente a marcas de persona y número) de las tres conjugaciones correspondientes a la pregunta 11 del apartado "Terminaciones de palabras" del cuestionario. La puntuación máxima podía ser de 36 puntos. Finalmente, la "Complejidad Gramatical" se obtuvo sumando las puntuaciones del apartado "Complejidad de frases" correspondientes a la forma Palabras y Oraciones. La puntuación máxima podía ser de 40 puntos.

Las pruebas estadísticas aplicadas son modelos de regresión. Se realizaron distintos modelos de regresión lineal y cuadrática simple en los que se utilizaron las variables anteriormente descritas. Cómo variable dependiente se seleccionó la morfología verbal, en un caso, y la complejidad gramatical en el otro. Cómo variables independientes se fueron introduciendo para cada uno de los modelos la edad, la masa léxica, la masa léxica nominal, la masa léxica verbal y la masa léxica de la categoría cerrada. En todos los casos los datos son puntuaciones directas. Por tanto, en total se realizaron 20 modelos de regresión diferentes en los que siempre se introducía una sola variable independiente y una sola variable dependiente con el objetivo de conocer el valor predictivo de una variable sobre la otra. Por esta razón todos los modelos de regresión se hicieron con un método de sólo un paso, bien con una estimación lineal en la mitad de ellas, bien con una estimación cuadrática en la otra mitad. Se usaron dos tipos de modelos con el objetivo de conocer si la relación entre las diferentes variables respondía a un modelo lineal, es decir, donde la varia- 
ble independiente produce aumentos progresivos en la variable dependiente o bien responde a un modelo cuadrático en el que existe una inflexión en un punto de la variable independiente que hace que se dispare el desarrollo de la variable dependiente. En todos los casos se cumplían los supuestos para la aplicación de los modelos de regresión dado que las variables son independientes entre ellas, existe normalidad en la distribución y los errores tienen variancia constante (homoscedasticidad).

\section{Resultados}

Los resultados se presentan en dos partes. En la primera de ellas se muestran los datos generales sobre el desarrollo de la morfología verbal y de la complejidad gramatical en relación con la edad. En la segunda parte se presentan específicamente datos sobre la interrelación entre el desarrollo léxico y el morfosintáctico. En este apartado se relaciona la cantidad de vocabulario con la competencia morfológica y complejidad sintáctica de las producciones infantiles. También se presentan resultados acerca del mejor predictor del desarrollo morfosintáctico. En primer lugar, se presentan resultados de la capacidad predictiva de las masas léxicas totales, nominales, verbales y clase cerrada respecto a la morfología verbal. En segundo lugar, se presentan los resultados de la capacidad predictiva de las masas léxicas totales, nominales, verbales y clase cerrada respecto a la complejidad gramatical.

\section{Edad y desarrollo morfosintáctico}

Los datos que muestran la relación de la edad con el desarrollo morfológico y con el gramatical se presentan en las figuras 1 y 2 .

FIGURA 1

Edad y morfología verbal

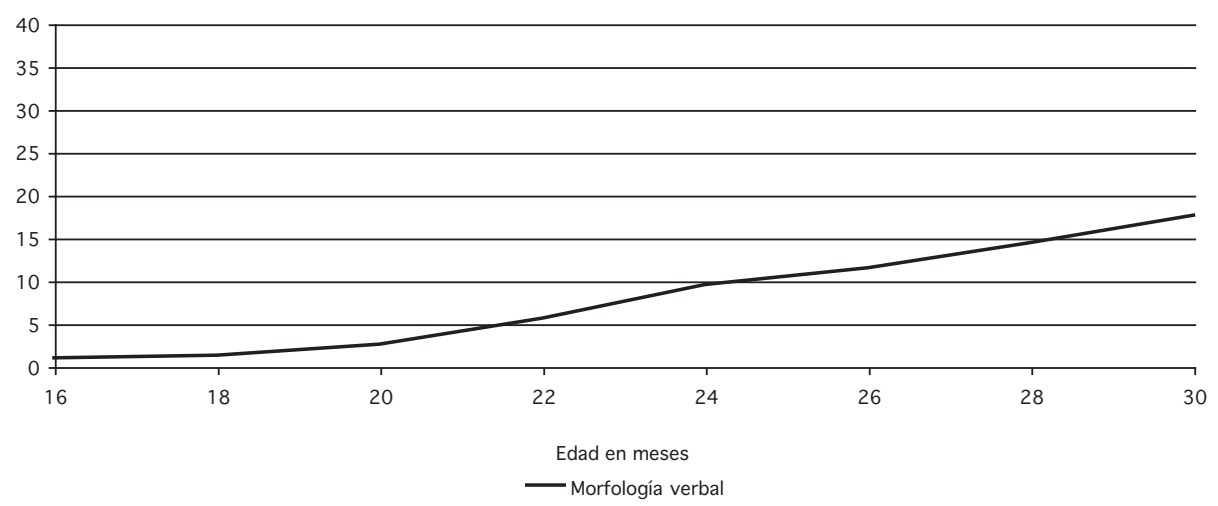

Como puede observarse, tanto la complejidad gramatical como la morfología verbal de tiempo y persona siguen un curso ascendente y progresivo que muestra un desarrollo mínimo hasta los 20 meses para, posteriormente, iniciar un progreso sostenido hasta los 30 meses. En el caso del desarrollo gramatical aparece un cambio notable alrededor de los 23 meses de edad, aunque también podemos observar otro cambio de tendencia hacia los 28 meses. Los datos de los modelos de regresión efectuados muestran que la edad es un predictor muy moderado del desarrollo morfológico verbal y del gramatical (ver Tabla II) ya que sólo permite explicar un $45 \%$ de la variancia, en el primer caso, en el modelo lineal y un $38 \%$, en el segundo, también en el modelo lineal. En el caso de los modelos cuadráticos, la edad permite explicar prácticamente lo mismo: $46 \%$ en el caso de la morfología y 39,8\% en el caso de la gramática. 
FIGURA 2

Edad y complejidad gramatical

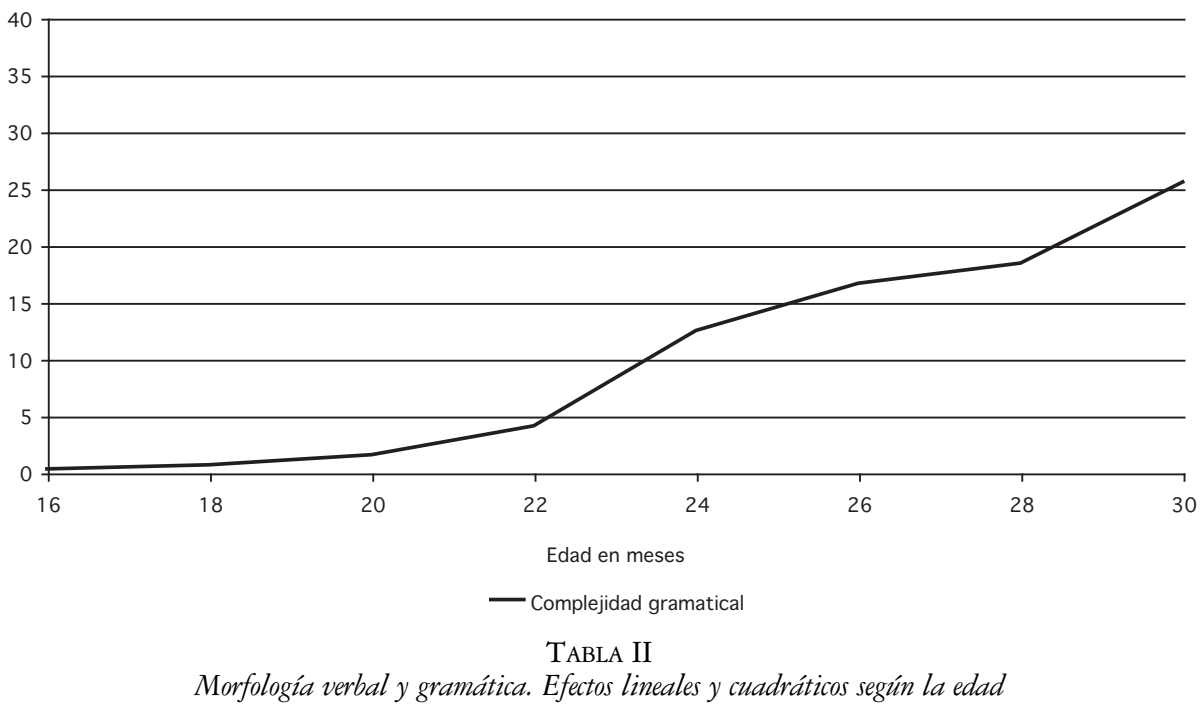

\begin{tabular}{lllll}
\hline & \multicolumn{2}{c}{ Lineal } & \multicolumn{2}{c}{ Cuadrático } \\
\hline Edad y morfología & $F=406,7 * * *$ & $R^{2}=0,451$ & $F=210,6 * * *$ & $R^{2}=0.460$ \\
Edad y gramática & $F=317,3 * * *$ & $R^{2}=0,381$ & $F=169,9 * * *$ & $R^{2}=0,398$ \\
\hline
\end{tabular}

$* * * p<0.001$

\section{Cantidad de vocabulario y desarrollo morfosintáctico}

En la tabla III se muestran los resultados de los diversos modelos de regresión lineal y cuadrático efectuados para comprobar si existe relación entre el desarrollo léxico y el inicio de la morfología verbal, así como para ver cual es el mejor predictor y qué modelo matemático sigue. La variable dependiente es la morfología verbal y las variables independientes, para cada uno de las modelos de regresión: la masa léxica general, la masa léxica nominal, la masa léxica verbal y la masa léxica de clase cerrada. Para estos análisis se utilizaron las variables sin agrupar, aunque, como se verá posteriormente, para la representación de las figuras, y con el fin de resumir y mostrar mejor los datos, los niños fueron agrupados en diferentes niveles en función de las diferentes masas léxicas.

TABLA III

Vocabulario y morfología verbal. Efectos lineales y cuadráticos según el tipo de masa léxica

\begin{tabular}{lllll}
\hline Masa léxica & \multicolumn{2}{c}{ Lineal } & \multicolumn{2}{c}{ Cuadrático } \\
\hline Total & $F=1399,6 * * *$ & $R^{2}=0,739$ & $F=751.4 * * *$ & $R^{2}=0.753$ \\
Nominal & $F=951,0$ & $R^{2}=0,658$ & $F=492,0$ & $R^{2}=0,666$ \\
Verbal & $F=1201,2 * * *$ & $R^{2}=0,708$ & $F=601,14 * * *$ & $R^{2}=0,709$ \\
Clase cerrada & $F=1773,2 * * *$ & $R^{2}=0,782$ & $F=941,7 * * *$ & $R^{2}=0,792$ \\
\hline
\end{tabular}

$* * * p<0.001$

En la tabla III puede apreciarse que ambos tipos de modelos de regresión, el lineal y el cuadrático, explican prácticamente la misma variancia del desarrollo de la morfología verbal. Por tanto, es preferible optar por el modelo lineal que es más simple, matemáticamente hablando. Todos estos valores permiten afirmar que las diferentes masas léxicas 
explican gran parte del desarrollo de la morfología verbal dado que todas estas variables son significativas y tienen valores superiores al $50 \%$. Sin embargo, de todas las variables independientes la que aparece como más explicativa es la masa léxica de clase cerrada (78\% de la variancia), seguida de la masa léxica total (73\% de la variancia) y de la masa léxica verbal (70\% de la variancia), finalmente la masa léxica que menos explica la morfología verbal es la nominal (65\% de la variancia).

FIGURA 3

Vocabulario total y morfología verbal

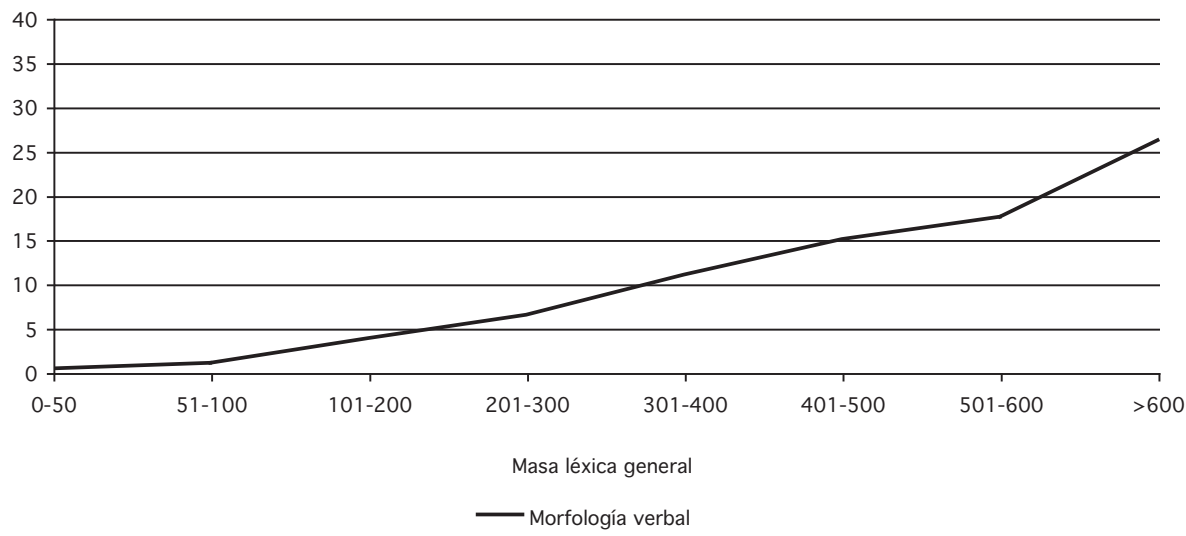

En la figura 3 puede observarse la media del desarrollo de la morfología verbal según la masa léxica general. Se observa que hasta una masa léxica de más de 100 palabras no se da prácticamente ningún contraste en las formas temporales. En cambio, a partir de las 200 palabras puede observarse un incremento que indica el inicio de la diversificación morfológica. También cabe destacar que a partir las 400-500 palabras el aumento se sucede con un progreso que se acerca a la puntuación máxima posible.

FigurA 4

Vocabulario de clase cerrada y morfología verbal

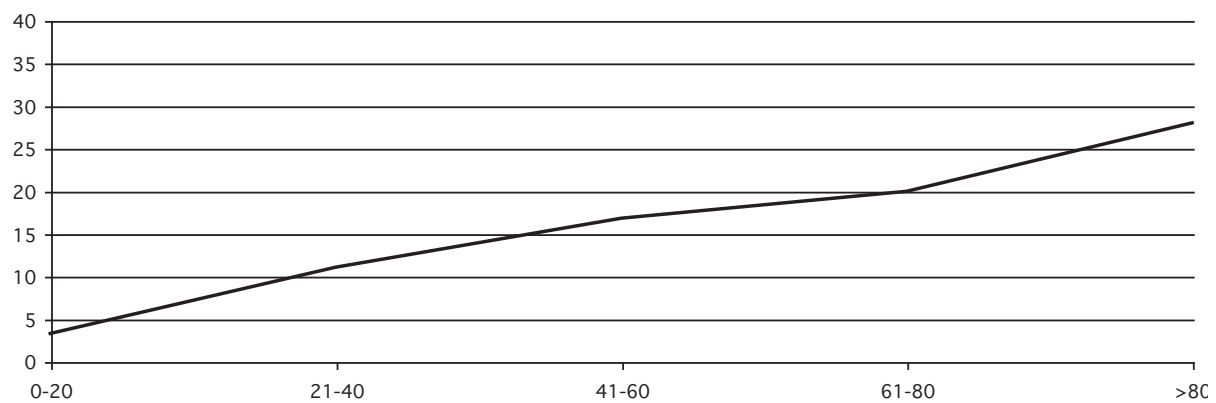

Masa léxica clase cerrada

Morfología verbal

Si tomamos como referencia la cantidad de vocabulario de clase cerrada para explicar el desarrollo de la morfología verbal (Figura 4), podemos observar que la diversificación morfológica se inicia ya con menos de 20 palabras de esta categoría, tiene un ascenso sostenido hasta las 61-80 palabras y da un salto después de las 80 palabras (aunque hay que tener en cuenta que el valor máximo de palabras de clase cerrada es de 90). 
En cuanto a la relación entre la adquisición de vocabulario y el desarrollo gramatical, en la tabla IV se ofrecen los datos al respecto. Se han realizado, también en este caso, modelos de regresión, lineal y cuadrática, tomando como variable dependiente la complejidad gramatical y como variables independientes los distintos tipos de vocabulario para cada uno de los modelos.

TABLA IV

Vocabulario y complejidad gramatical. Efectos lineales y cuadráticos según el tipo de masa léxica

\begin{tabular}{lllll}
\hline Masa léxica & \multicolumn{2}{c}{ Lineal } & \multicolumn{2}{c}{ Cuadrático } \\
\hline Total & $F=1088,7 * * *$ & $R^{2}=0,679$ & $F=661,0 * * *$ & $R^{2}=0,720$ \\
Nominal & $F=813,0$ & $R^{2}=0,612$ & $F=476,6$ & $R^{2}=0,650$ \\
Verbal & $F=1001,29 * * *$ & $R^{2}=0,660$ & $F=519,0 * * *$ & $R^{2}=0,669$ \\
Clase cerrada & $F=1255,1 * * *$ & $R^{2}=0,709$ & $F=664,3 * * *$ & $R^{2}=0,721$ \\
\hline
\end{tabular}

$* * * p<0.001$

Como puede observarse, en alguna de las relaciones efectuadas, el modelo cuadrático explica ligeramente una mayor proporción de variancia que el modelo lineal, por lo que podemos decir que, no sólo existe un crecimiento de la complejidad a medida que aumenta el vocabulario, sino que el aumento es proporcionalmente mayor a partir de un punto, dado que la relación entre ambas variables se ajusta más a una curva que no a una línea recta. Como en el caso anterior, todos estos valores permiten afirmar que las diferentes masas léxicas explican gran parte del desarrollo de la gramática dado que todas estas variables son significativas y tienen valores superiores al $50 \%$. Aunque, también en este caso, de todas las variables independientes las más explicativas vuelven a ser la masa léxica de categoría cerrada (72\%) junto con la masa léxica general (72\%), seguidamente se encuentran la masa léxica verbal (66\% de la variancia) y la masa léxica nominal (65\% de la variancia).

En las figuras 5 y 6 se visualiza esta relación entre la cantidad de vocabulario y el desarrollo gramatical.

FIGURA 5

Vocabulario total y complejidad gramatical

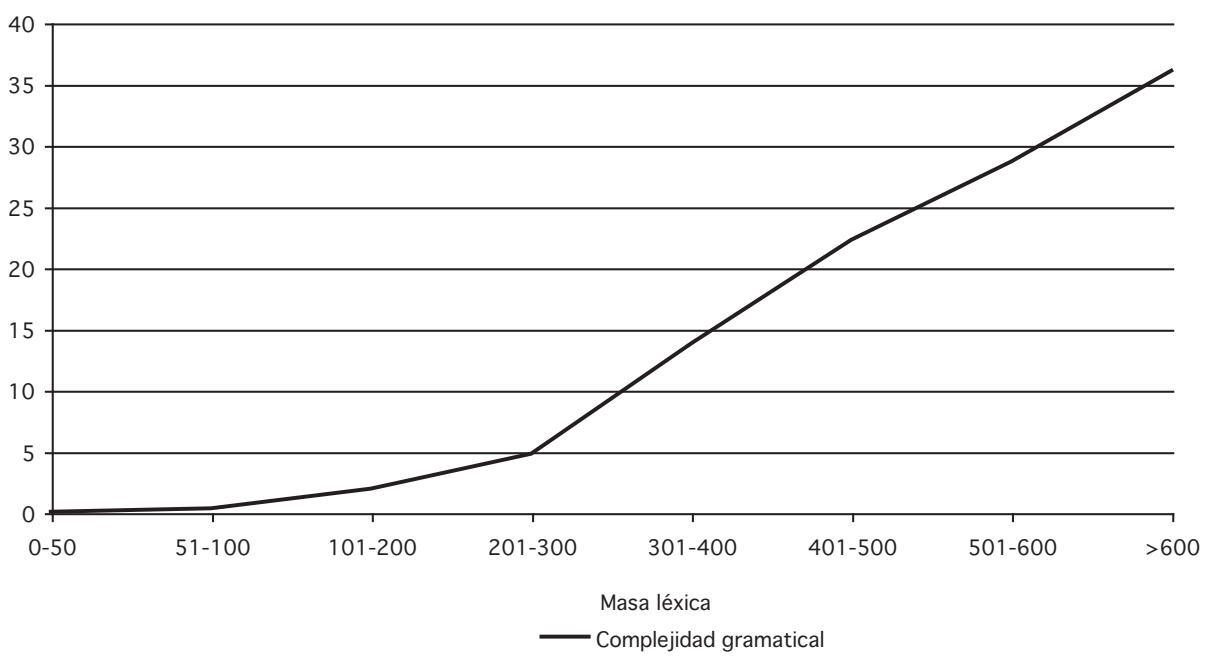

La complejidad sintáctica comienza a manifestarse de manera más notoria a partir de las 200-300 palabras -vocabulario general (ver Figura 5). A partir de esa cantidad de 
FIGURA 6

Vocabulario clase cerrada y complejidad gramatical

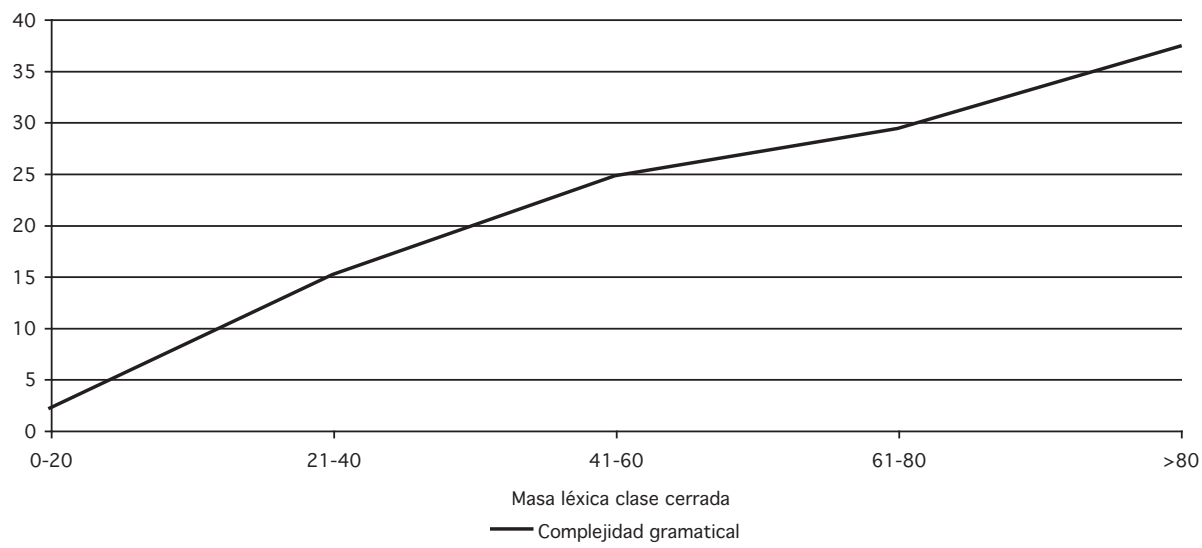

palabras el aumento de la complejidad sintáctica se manifiesta progresivo y relevante. Hay que tener en cuenta que en el caso del vocabulario general el modelo cuadrático explica, aunque muy ligeramente (4\%), una mayor proporción de variancia que el modelo lineal. Por esta razón, la curva que mejor explica el desarrollo tiene un punto de inflexión, que en este caso y observando la figura 5, se sitúa en las 300 palabras de la masa léxica total. En el caso de la clase léxica cerrada, la complejidad sintáctica empieza a mostrarse prácticamente desde el inicio (0-20) y mantiene un desarrollo sostenido hasta el máximo de esta clase (ver Figura 6).

\section{Discusión}

Los resultados han mostrado como la morfología verbal se adquiere muy gradualmente al inicio hasta los 22-24 meses y de manera más rápida a partir de estos meses. Un patrón parecido muestra la complejidad gramatical, aunque con una evolución ligeramente menos lineal y con un salto más abrupto hacia los 24 meses. Por tanto, de manera general, podemos situar cierta explosión o aceleración morfosintáctica a partir de los dos años de edad. Estos resultados son compatibles con las descripciones acerca del desarrollo verbal y sintáctico en lengua castellana y catalana (Serra, Serrat, Solé, Bel y Aparici, 2000; entre otros).

Por otra parte, los resultados hallados nos muestran que la cantidad de vocabulario es mejor predictor del desarrollo morfosintáctico que la edad, como ya habían apuntado otros autores (Marchman y Bates, 1994). Este dato avala las propuestas presentadas anteriormente acerca de la fuerte relación entre el aprendizaje del léxico y el desarrollo morfosintáctico. De hecho, se aprecia de manera muy importante que los distintos tipos de masa léxica examinados son todos ellos mucho mejores predictores de este desarrollo que la edad de los sujetos. Ahora bien, hay dos tipos de vocabulario que destacan por situarse como los mejores predictores del desarrollo, tanto de la morfología verbal como de la complejidad gramatical. Se trata del vocabulario general y del vocabulario de clase cerrada. Para comprender e intentar explicar este hecho hay que tener en cuenta que el vocabulario general incluye, además del vocabulario nominal y del verbal, la clase cerrada de palabras, donde se hallan las palabras funcionales, como preposiciones, conjunciones, determinantes y otros. Por tanto, atendiendo a los datos puede considerarse que el vocabulario de clase cerrada destaca como un buen predictor del desarrollo morfológico verbal y del desarrollo gramatical.

Esta evidencia parece clara en el caso del desarrollo gramatical puesto que las palabras incluidas en la clase cerrada (por ejemplo, los nexos) son necesarias para construir 
enunciados progresivamente más complejos. Sin embargo, que el vocabulario de clase cerrada sea también más explicativo del desarrollo de la morfología verbal, no resulta tan fácilmente explicable. Consideramos interesante profundizar en esta relación ya que ello permitiría predecir mejor el desarrollo gramatical a partir de aprendizajes previos y, en definitiva, evaluar mejor a los niños, tanto de desarrollo lingüístico normal como de desarrollo alterado. De hecho, esta relación que se ha hallado entre el léxico de clase cerrada y el desarrollo morfosintáctico permite considerar que los ítems de este tipo de los cuestionarios MacArthur CDI-II pueden utilizarse como elementos clave para predecir el desarrollo gramatical posterior.

Como apuntábamos en la introducción, nuestro estudio seguía la sugerencia de algunos autores, fundamentalmente Bates y Goodman (1997) y Dixon y Marchman (2007) quienes consideraban interesante ampliar la investigación sobre la influencia de la cantidad de vocabulario en los aspectos gramaticales, para centrarla en 'masas léxicas' específicas, más allá de medidas léxicas globales. Si bien esta sugerencia ha resultado interesante en cuanto al papel del vocabulario de clase cerrada, los resultados de nuestro estudio señalan que el vocabulario específico no siempre es mejor predictor del aprendizaje de los aspectos morfosintácticos asociados al mismo. Así, de los resultados se desprende que, tanto en el caso de la complejidad gramatical como en el caso de la morfología verbal, existe una relación con la masa léxica verbal y ésta explica un alto porcentaje de la variancia (entre el $66 \%$ y el $70 \%$ ), aunque este porcentaje no es superior al del vocabulario general o del vocabulario de clase cerrada, ya que explica un poco menos de la variancia observada que aquéllos. En otras palabras, en el caso de la masa léxica verbal, nuestros datos muestran que este vocabulario no es más explicativo del aprendizaje de la morfología verbal que el vocabulario general -que lo incluye- o que el vocabulario de clase cerrada.

En cuanto al tipo de relación entre vocabulario y desarrollo morfosintáctico, en los datos que presentamos no hallamos una fuerte evidencia de una relación no lineal entre el desarrollo del léxico y el de los aspectos morfosintácticos analizados. Así pues, nuestro trabajo se sitúa en la línea de las sugerencias de Dixon y Marchman (2007), y de los resultados de Anisfeld et al. (1998) o Serrat et al. (2004). Solamente en el caso del vocabulario general, y en relación con el aprendizaje gramatical, se observa un inicio hacia las 200 palabras de la complejidad gramatical y un salto diferencial que se da hacia las 300-400 palabras. Este cambio, que se ha referido en otros estudios como en Bates y Goodman (1997), en Serra y Sanz-Torrent (2004), podría interpretarse, según Dixon y Marchman (2007), atendiendo a aspectos relacionados con la recogida de datos.

En realidad, tal y como también se observa en relación con el desarrollo de la morfología y la complejidad sintáctica por edad, parece que siempre se da una relación claramente lineal en el caso del desarrollo morfológico, en cambio la complejidad sintáctica sufre cambios en la adquisición que concuerdan algo más con un modelo cuadrático. Los resultados de Marchman et al. (2004) también evidencian, tanto en español como en inglés, una relación no lineal entre vocabulario general y complejidad gramatical (Figuras 1 y 2 en Marchman et al., 2004). Por tanto, aunque muy ligeramente, al examinar la relación con alguno de los tipos de léxico, observamos que el desarrollo sintáctico presenta un cambio de tendencia a partir de una cierta cantidad de vocabulario o 'masa crítica'. Esta cantidad 'crítica' de vocabulario sería necesaria para producir un nivel particular de complejidad gramatical. De nuevo, es importante resaltar que datos de este tipo pueden resultar interesantes con respecto a situaciones de evaluación del desarrollo lingüístico.

En definitiva, los datos indican una relación estrecha entre distintos tipos de vocabulario, incluso en el caso del vocabulario nominal, y el proceso de gramaticalización. Dicha relación, en cuanto al desarrollo morfológico se presenta fundamentalmente de forma lineal, mientras que el desarrollo gramatical parece evidenciar, aunque muy ligeramente, un cambio de tendencia a partir de cierta cantidad de vocabulario. Como 
sugieren los resultados de Stolt et al. (2009) diferentes estructuras gramaticales muestran diferentes grados y tipos de dependencia léxica.

La relación hallada entre el componente léxico y el morfológico y/o gramatical puede interpretarse aduciendo que es necesaria una interdependencia mutua entre ambos componentes para el desarrollo del lenguaje durante la infancia, aunque también puede considerarse que la influencia entre ambos componentes se da de maneras diferentes en diferentes momentos del desarrollo. A nuestro entender, no estamos ante una bidireccionalidad simple según la cual el vocabulario influye en la gramática y, a su vez, la gramática lo hace en el léxico a lo largo de todo el desarrollo, sino que en ciertos momentos del desarrollo determinada cantidad de vocabulario -o determinado tipo de vocabulario- constituye una base necesaria para el inicio de la gramática. De hecho, varios de los estudios que hemos tratado aquí muestran que esta dirección de la influencia cobra relevancia en los inicios gramaticales. Por otro lado, algunos autores como Dione, Dale, Boivin y Plomin (2003) aportan evidencia sobre una influencia bidireccional en edades más avanzadas, hacia los 3 o 4 años. Cómo ocurre exactamente, qué componentes se hallan implicados y en qué momentos del desarrollo, son cuestiones para profundizar en futuros estudios.

\section{Referencias}

AKHTAR, N. (1999). Acquiring basic word order: Evidence for data-driven learning of syntactic structure. Journal of Child Language, $26(2), 339-356$.

Anisfeld, M., RosenberG, E. S., Hoberman, M. J. \& Gasparini, D. (1998). Lexical acceleration coincides with the onset of combinatorial speech. First Language, 18, 165-184.

Barreña, A., García, I., Ezeizabarrena, M. J., Almgren, M., Arratibel, N., Olano, I., Barnes, J., Petuya, A. \& ColiNA, A. (2008). MacArthur-Bates komunikazio garapena neurtzeko zerrenda. Euskarara egokitua. Erabiltzaileentzako gida eta eskuliburu teknikoa. Bilbao: UEU

BASSANO, D. (2000). Early development of nouns and verbs in French: Exploring the interface between lexicon and grammar. Journal of Child Language, 27, 521-559.

BATES, E., BRETHERTON, I. \& SNYDER, L. (1988). From first words to grammar: Individual differences and dissociable mechanisms. Cambridge: CUP

BATES, E. \& GOODMAN, J. C. (1997). On the inseparability of grammar and the lexicon: Evidence from acquisition, aphasia and real-time processing. En G. Altmann (Ed.), Special issue on the lexicon, Language and Cognitive Processes, 12 (5/6), 507584.

Bowerman, M. (1988). The «No Negative Evidence» Problem: How Do Children Avoid Constructing an Overly General Grammar. En J. Hawkins (Ed.), Explaining Linguistic Universals (pp. 73-101). Oxford: Blackwell.

Braine, M. D. (1976). Children's first word combinations. Monographs of the Society for Research in Child Development, 41 (1), 104-105.

Caselli, M. C., Casadio, P. \& Bates E. (1999). A comparison of the transition from first words to grammar in English and Italian. Journal of Child Language, 26, 69-111.

Conboy, B. T. \& ThaL, D. (2006). Ties between the lexicon and grammar: Cross-sectional and longitudinal studies of bilingual toddlers. Child Development, 77 (3), $712-735$.

Dionne, G., Dale, P. S., BoIvin, M. \& Plomin, R. (2003). Genetic evidence for bidirectional effects of early lexical and grammatical development. Child Development, 74 (2), 394-412.

Dixon, J. A. \& Marchman, V. A. (2007). Grammar and the Lexicon: Developmental Ordering in Language Acquisition. Child Development, $78(1), 190-212$.

DromI, E. (1987). Early lexical development. Londres: Cambridge University Press.

Fenson, L. P., Dale, J. S., Reznick, D., Thal, E., Bates, J. P., Hartung, S., Pethick, J. \& Reilly, J. (1993). MacArthur Communicative Development Inventories: User's guide and technical manual. Baltimore: Brookes.

Fenson, L., Marchman, V. A., Thal, D., Dale, P. S., Reznick, J. S. \& Bates, E. (2007). The MacArthur Communicative Development Inventories: User's guide and technical manual. $2^{\mathrm{a}}$ Ed. Baltimore: Paul H. Brooks.

Fisher, C., Hall, G., Rakowitz, S. \& Gleitman, L. (1994). When it is better to receive than to give: Syntactic and conceptual constraints on vocabulary growth. Lingua. 92, 333-375.

Galeote, M. A., Soto, P., Serrano, A., Pulido, L., Rey, R. \& Martínez-Roca, R. (2006). Un nuevo instrumento para evaluar el desarrollo comunicativo y lingüístico de niños con síndrome de Down. Revista Española de Información e Investigación sobre el sindrome de Down, 20-26.

García, I., Arratibel, N., Barreña, A. \& Ezeizabarrena, M. J. (2008). Adaptación de los inventarios MacArthur-Bates al euskara: desarrollo comunicativo entre los 8 y 30 meses. Infancia y Aprendizaje, 31 (4), 411-424.

Gleitman, L. (1990). The structural sources of verb meanings. Language Acquisition. 1, 3-55.

GOLDBERG, A. (1995). Constructions: a construction grammar approach to argument structure. Chicago, IL: The University of Chicago Press.

GoldBERG, A. (1999). The Emergence of Argument Structure Semantics. En B. MacWhinney (Ed.), The Emergence of Language (pp. 197-212). Hillsdale: Lawrence Erlbaum Publications.

Goldberg, A. E., Casenhiser, D. M. \& Sethuraman, N. (2004). Learning argument structure generalizations. Cognitive Linguistics, 15 (3), 289-316. 
Grimshaw, J. (1981). Form, function, and the language acquisition device. En C. L. Baker \& J. McCarthy (Eds.), The Logical Problem of Language Acquisition (pp. 165-182). Cambridge: MIT Press.

Jackson-Maldonado, D., Thal, D., Fenson, L., Marchman, V., Bates, E. \& Gutiérrez-Clellen, V. (1993). Early lexical development in Spanish-speaking infants and toddlers. Journal of Child Language, 20, 523-549.

Lieven, E., PINE, J. \& BALDWIN, G. (1997). Lexically-based learning and early grammatical development, Journal of Child Language, 24, 187-219.

lópez Ornat, S., Gallego, C., Gallo, P., Karousou, A., Mariscal, S. \& Martínez, M. (2005). Inventarios de Desarrollo Comunicativo MacArthur: Manual Técnico. Madrid: Ediciones TEA.

Maital, S. L., Dromi, E., Sagi, A. \& Bornstein, M. H. (2000). The Hebrew Communicative Development Inventory: language specific properties and cross-linguistic generalitzations. Journal of Child Language, 27, 43-67.

Marchman, V. A. \& Bates, E. (1994). Continuity in lexical and morphological development: a test of the critical mass hypothesis. Journal of child language, 21,339-66.

Marchman, V. A., MartíneZ-SussmanN, C. \& Dale, P. S. (2004). The language-specific nature of grammatical development: Evidence from bilingual language learners. Developmental Science, 7 (2), 212-224.

NAIGLES, L. (1996). The use of multiple frames in verblearning via syntactic bootstrapping. Cognition, 58, 221-251.

NAIGLES, L. \& HofF-GinsBerg, E. (1995). Input to verb learning: evidence for the plausibility of syntactic bootstrapping. Developmental Psychology, 31, 827-837.

PéreZ PEREIRA, M. \& GARCíA SOTO, X. R. (2003). El diagnóstico del desarrollo comunicativo en la primera infancia: Adaptación de las escalas MacArthur al gallego. Psicothema, 15 (3), 352-361.

Pérez Pereira, M. \& Resches, M. (2007). Elaboración de las formas breves del "Inventario do Desenvolvemento de Habilidades Comunicativas": datos normativos y propiedades psicométricas. Infancia y Aprendizaje, 30 (4), 565-588.

Pérez Pereira, M. \& ResChes, M. (2008). Validez concurrente y predictiva del IDHC. Implicaciones para el posterior desarrollo del lenguaje y de la cognición social. En E. Díez-Itza (Ed.), Estudios de desarrollo del lenguaje y educación (pp. 87-94). Oviedo: Universidad de Oviedo ICE.

PINKER, S. (1984). Language learnability and language development. Cambridge, MA: Harvard University Press.

PinKer, S. (1989). Language Acquisition. En D. N. Osherson \& H. Lasnik (Eds.), An invitation to cognitive science; Volume1 Language (pp. 199-241). Cambridge, MA: MIT Press.

Plunkett, K. \& Marchman, V. (1993). From rote learning to system building: Acquiring verb morphology in children and connectionist nets. Cognition, 48 (1), 21-69.

Serra, M. (2008). Las interfaces entre los componentes del lenguaje: Tierra de nadie en un viaje obligatorio. En E. Diaz-Itza (Ed.), Estudios de desarrollo del Lenguaje y Educación (pp. 511-51). Oviedo: Publicaciones de la Universidad de Oviedo.

SERra, M. \& SANZ-Torrent, M. (2004). "Las 400 palabras": datos para una perspectiva constructivista de la interfaz léxicosintáctica. Anuario de Psicología, 35 (2), 235-255.

Serra, M., Serrat, E., Solé, R., Bel, A. \& Aparici, M. (2000). La adquisición del lenguaje. Barcelona: Ariel.

Serrat, E., Olmo, R., Badia, I., Sanz-Torrent, M., Aguilar, E., Lara, M. \& Serra, M. (2005). The Catalan version of the MacArtbur-Bates scales (CDI-II). Poster presentado en Xth International Congress For the Study of Child Language. Berlín.

Serrat, E., Sanz-Torrent, M. \& Bel, A. (2004). Aprendizaje léxico y desarrollo de la gramática: vocabulario verbal, aceleración morfológica y complejidad sintáctica. Anuario de Psicología, 35 (2), 221-234.

Stolt, S., HaAtaja, L., Lapinleimu, H. \& Lehtonen, L. (2009). Associations between lexicon and grammar at the end of the second year in Finnish children. Journal of Child Language, 36, 779-806

ThaL, D., JaCKSON MALDONADO, D. \& ACOSTA, D. (2000). Validity of a parent-report measure of vocabulary and grammar for spanish-speaking toddlers, Journal of Speech. Language and Hearing Research, 43, 1087-1100

THORDARdotTIR, E., ElLIS WeISMER, S. \& EvANS, J. (2002). Continuity in lexical and morphological development in Icelandic and English-speaking 2 years-old. First Language, 22, 3-28.

Tomasello, M. (2003). Constructing a Language: A Usage-Based Theory of Language Acquisition. Cambridge, MA: Harvard University Press. 